\title{
Diagnóstico de la superación profesional técnica de los profesores de la rama eléctrica
}

\section{Diagnosis of the technical professional improvement of the teachers of the electric branch}

\author{
Zeidy Sandra López Collazo \\ Universidad de Artemisa, Cuba. Código Postal: 33700
}

Recibido el 14 de junio del 2017, aceptado el 28 de diciembre del 2017

DOI: https://doi.org/10.33017/RevECIPeru2017.0016/

\section{Resumen}

El trabajo presenta el diagnóstico del estado inicial de la superación profesional técnica de los profesores de la rama eléctrica de los institutos politécnicos en la provincia de Artemisa, Cuba. El objetivo consistió en caracterizar la superación profesional técnica de los profesores de la rama eléctrica de los institutos politécnicos en la provincia de Artemisa a partir de su definición y la determinación de dimensiones e indicadores. Para ello se procedió a la búsqueda bibliográfica que posibilitó la elaboración de la definición de la superación profesional técnica de los profesores de la rama eléctrica y su operacionalización en dimensiones e indicadores, los que fueron presentados en diferentes escenarios, todo lo cual tributó a su rediseño y perfeccionamiento. Se utilizaron diferentes métodos del nivel teórico, empírico y métodos estadísticos que permitieron realizar el diagnóstico de este proceso y precisar las principales fortalezas y limitaciones. Las tendencias centrales del estado de las dimensiones se ubican en el rango evaluativo de bajo, alejado de la tendencia central: la dimensión cognitiva $(1,9)$; la dimensión procesual $(1,99)$ y la dimensión actitudinal (2,75). Se reconocen insuficiencias en la articulación interinstitucional para la actualización, complementación y reorientación del contenido técnico profesional de los profesores de la rama eléctrica, pues no se aprovechan las potencialidades del entrenamiento como forma de organización. El coeficiente de correlación multidimensional rpj se comportó para las dimensiones en los criterios de (altamente coherente), los cuales permiten inferir con un alto grado de probabilidad, que se realizaron análisis objetivos y por tanto, confiables.

Descriptores: superación profesional técnica, dimensiones e indicadores

\section{Abstract}

The work presents the diagnosis of the initial state of the technical professional improvement of the teachers of the electrical branch of the polytechnic institutes in the province of Artemisa, Cuba. The objective was to characterize the technical professional improvement of the professors of the electrical branch of the polytechnic institutes in the province of Artemisa based on their definition and the determination of dimensions and indicators. To do this, we proceeded to the bibliographic search that made possible the elaboration of the definition of the technical professional improvement of the professors of the electric branch and its operationalization in dimensions and indicators, which were presented in different scenarios, all of which contributed to its redesign and improvement. Different methods were used at the theoretical, empirical and statistical methods that allowed to diagnose this process and to specify the main strengths and limitations. The central tendencies of the state of the dimensions are located in the low evaluative range, far from the central tendency: the cognitive dimension (1,9); the processual dimension (1.99) and the attitudinal dimension (2.75). 
Weaknesses are recognized in the interinstitutional articulation for the updating, complementation and reorientation of the professional technical content of the professors of the electrical branch, because the potentialities of training as a form of organization are not taken advantage of. The multidimensional correlation coefficient rpj behaved for the dimensions in the (highly coherent) criteria, which allow us to infer with a high degree of probability, that objective and, therefore, reliable analyzes were

\section{Keywords: technical professional development, dimensions and indicators}

\section{Introducción}

La Revolución Científico-Técnica provoca constantes cambios en la ciencia y la tecnología, que se ponen en función del desarrollo de la industria, de la producción y los servicios. Para aplicarlos es preciso acudir a la educación y así actualizar los conocimientos y habilidades de los hombres para llevar adelante el desarrollo de sus países.

La superación profesional toma un papel cada vez más protagónico en la sociedad actual, pues los conocimientos, al menos los relacionados con la especialidad, requieren de una actualización constante. Es por ello que hoy día se ha reclama como algo indispensable para el desarrollo profesional en cualquier ámbito laboral. Desde que se introdujo en la bibliografía especializada el término de aprendizaje a lo largo de la vida en la Educación Técnica y Profesional (ETP), la superación profesional entró en una nueva etapa de conceptualización e importancia. Enseñar no enseña a enseñar sino que se necesita una base que se vaya reciclando en la medida en que los contenidos y la propia sociedad cambian.

Desde entonces hasta la actualidad, el concepto de superación profesional del profesorado de la ETP se ha consolidado con fuerza en el panorama educativo; cada vez más, la comunidad educativa está consciente de la necesidad de reciclarse continuamente, de superarse para mejorar la propia práctica y de la innovación como propuesta de cambio; en definitiva, se trata de estar dispuestos a aprender con la finalidad de conseguir un verdadero desarrollo profesional y una calidad de la docencia de su especialidad, bajo la concepción de superación profesional técnica.

En la búsqueda de una respuesta a esta problemática se ha trabajado en definir la superación profesional técnica de los profesores de la rama eléctrica, así como las dimensiones e indicadores que viabilizan su caracterización, lo que constituye el objetivo de este trabajo.

\section{Desarrollo}

La lógica investigativa se basa en el estudio del proceso de superación profesional técnica desde un punto de vista transformador y práctico al considerar los rasgos y aspectos limitantes que se manifiestan para su transformación, a partir de la vinculación de los métodos del nivel teórico con los del nivel empírico y estadísticos.

Métodos del nivel teórico: Analítico-sintético y sistematización.

Métodos del nivel empírico: Análisis documental, observación científica y encuesta.

Métodos estadísticos: Se utiliza la estadística descriptiva para procesar cuantitativamente los datos obtenidos mediante los instrumentos de los métodos del nivel empírico. Además, se utilizó la estadística inferencial a partir del empleo del coeficiente de correlación multidimensional rpj, mediante la aplicación del software de procesamiento Cohaerentîa y el procedimiento lógico de ruptura con la racionalidad y la lógica clásicas; para determinar la fiabilidad, el grado de coherencia y objetividad de los resultados.

Grupo de estudio: El grupo de estudio lo constituye un total de 20 profesores de la rama eléctrica del IP "Juan Manuel Castiñeiras García” del municipio Mariel, quienes se desempeñan en la familia de especialidades Eléctrica, 11 directivos y ocho funcionarios de las direcciones municipales y provinciales de la ETP.

\section{Resultados y su discusión}

Para cumplimentar el objetivo formulado se partió de una revisión bibliográfica que permitió definir la superación profesional técnica de los profesores de la rama eléctrica y luego en función de ello se procede a realizar su operacionalización para identificar las dimensiones e indicadores que expresen, con la mayor objetividad posible, los rasgos distintivos que permitan realizar su medición de forma empírica, cuantitativa y cualitativamente. 
La determinación de las dimensiones e indicadores tuvo una primera aproximación en los resultados derivados del Proyecto de Desarrollo "Impacto de las Transformaciones en el IP Mártires de Guanajay" en el año 2011, los cuales fueron aprobados por el Consejo Científico Territorial de la extinta Universidad de Ciencias Pedagógicas "Rubén Martínez Villena" (UCPRMV) y se materializó en: Estrategia que posibilite la integración del instituto politécnico con las entidades laborales y la comunidad.

Asimismo, tanto las dimensiones como los indicadores de la variable, han sido presentados en diferentes escenarios como fueron el Departamento de ETP de la UCPRMV, actual Departamento de Educación Laboral-Informática de la Universidad de Artemisa, con la participación de profesores con experiencia en la actividad práctica de las diferentes disciplinas, entre ellas Electrotecnia; el Departamento de Electricidad del instituto politécnico "Juan Manuel Castiñeiras" conjuntamente con especialistas de la Central Termoeléctrica (CTE) "Máximo Gómez" del municipio Mariel y en el Consejo Científico de la Facultad de Ciencias de la Educación Media de la Universidad de Artemisa con la presencia de Doctores en Ciencias Pedagógicas, quienes oportunamente ofrecieron criterios y sugerencias que permitieron su rediseño y perfeccionamiento con vistas a alcanzar en la superación profesional técnica niveles de actualización, complementación y reorientación del contenido técnico profesional de la rama eléctrica.

Luego de operacionalizada la variable, se realizó la parametrización de los indicadores atendiendo a la escala de: inadecuado (1), poco adecuado (2), adecuado (3), bastante adecuado (4) y muy adecuado (5).

Para caracterizar el estado inicial de la superación profesional técnica de los profesores de la rama eléctrica de los institutos politécnicos en la provincia de Artemisa, se aplicaron diferentes instrumentos correspondientes a la aplicación de los métodos empíricos declarados. Como datos generales se indagó acerca de los años de experiencia de los profesores de la rama eléctrica y del personal directivo encuestado.

El análisis documental se realizó a través de una guía de revisión documental y se estudiaron documentos oficiales del Ministerio de Educación (MINED) y del Ministerio de Educación Superior
(MES) relacionados con la superación profesional, entre ellos el balance de las transformaciones en la ETP, sus prioridades hasta el año 2013 y las estrategias de superación de la Dirección Provincial de Educación (DPE) de Artemisa, correspondiente a los cursos del 2011 al 2013.

Se aplicó una guía de observación al proceso de superación profesional, distribuidas en diez observaciones al desarrollo de talleres realizados a nivel del Departamento de Electricidad del instituto politécnico "Juan Manuel Castiñeiras" y dos cursos de superación realizados en la actual Universidad de Artemisa, que permitieron centrar la atención en aspectos que constituyen indicadores del proceso de superación profesional técnica de los profesores de la rama eléctrica.

Fueron encuestados once directivos de la ETP y a los veinte profesores de la rama eléctrica, diez de la especialidad de Electricidad y diez de la especialidad Electricidad de Mantenimiento Industrial.

Para sintetizar los resultados obtenidos en la observación realizada, se determinó el estado de cada indicador a partir del cálculo de la mediana (Me) para datos agrupados, de manera que pueda discriminarse con mayor precisión la tendencia central que caracteriza al escalamiento ordinal utilizado, de acuerdo con la fórmula [1].

$$
M_{d}=L_{0}+\frac{\frac{n}{2}-\sum f_{A M_{d}}}{f_{M_{d}}} C
$$

Para determinar el grado de coherencia y objetividad de los resultados obtenidos se aplicó el coeficiente de correlación multidimensional rpj, el cual establece los rangos de coherencia que permiten atribuir un criterio sobre la objetividad de las valoraciones realizadas, de acuerdo con la fórmula [2].

$$
r_{p j}=1-\frac{12 \sum_{p=1}^{n} \sum_{j=1}^{N} d_{p j}^{2}}{\left(n^{2}-n\right)\left(N^{3}-N\right)}
$$

Finalmente se realiza la triangulación de los instrumentos aplicados, la guía de observación, la encuesta a directivos y la encuesta a profesores, por considerar que "la triangulación entre métodos ofrece la oportunidad de mejorar el diagnóstico organizativo sintetizando los resultados derivados 
de la utilización de múltiples métodos científicos en una interpretación válida y coherente." [3].

\subsection{Acercamiento a la definición de la superación profesional técnica de los profesores de la rama eléctrica}

Se realizó la consulta bibliográfica a investigaciones que han incursionado en el estudio de esta categoría, tales como Fernández (2014) [4], quienes ofrecen valiosos aportes donde se vislumbra lo cognitivo, procedimental y axiológico para lograr cambios y transformaciones en la formación de la competencia técnico-profesional de los profesionales, a partir de la adquisición, ampliación y actualización continua del contenido técnico profesional en un proceso de enseñanzaaprendizaje, limitándose la intencionalidad del componente actitudinal y procesual, así como el alcance de la superación profesional técnica en torno a la apropiación, complementación y reorientación continua del contenido técnico profesional en un proceso pedagógico, desarrollado en un contexto educativo de la ETP como lo es la entidad laboral.

A partir de este análisis se defiende la idea de que la superación profesional técnica de los profesores de la rama eléctrica debe estar centrada en un proceso pedagógico de adquisición, apropiación, actualización, complementación y reorientación continuas del contenido técnico profesional de la especialidad, asociados al avance y el desarrollo científico técnico a partir de formas organizativas en el contexto de la entidad laboral. Por consiguiente, la autora define superación profesional técnica de los profesores de la rama eléctrica como "el proceso pedagógico, que facilita a los graduados universitarios, la adquisición, apropiación, actualización, complementación y reorientación continuas del contenido técnico profesional de la rama eléctrica, asociados al avance y el desarrollo científico técnico, a partir de formas organizativas en el contexto de la entidad laboral." [5].

En el proceso de superación profesional técnica en la rama eléctrica como en cualquier otra especialidad técnica, la esfera cognitiva, está estrechamente relacionada con la afectiva motivacional, lo que implica que se debe explorar la personalidad en su integridad y no solamente sus conocimientos, hábitos y habilidades. Cuando los objetivos se vinculan con los motivos, la superación profesional técnica adquiere un fuerte sentido personal, que contribuye a su exitosa realización. Si por el contrario, no existe relación entre las motivaciones del profesor y los objetivos que se ha proyectado alcanzar, esta superación carece de sentido, lo que afecta su ejecución. En cuanto a las actitudes son las que revelan el sentir y la convivencia del profesor en la entidad laboral, el saber actuar dentro de escenarios definidos.

\subsection{Dimensiones e indicadores de la superación profesional técnica de los profesores de la rama eléctrica}

Desde el punto de vista teórico se asume como dimensión e indicador las definiciones ofrecidas por Pérez (2011) al plantear que dimensión: "son los rasgos que facilitarán una primera división dentro del concepto. Es decir, son diferentes partes a analizar, o simplemente diferentes direcciones del análisis del proceso" y como indicador: "conjunto de rasgos perceptibles que harán posible una referencia empírica a la presencia del concepto, es la manifestación externa de la característica 0 el atributo, con posibilidad incuestionable de ser registrado." [6]

A tono con el autor, y por la relación que guarda con la definición de superación profesional técnica de los profesores de la rama eléctrica, se influye en las esferas cognitiva y volitivo-afectiva-motivacional a partir del contenido técnico profesional. Para ello se determinaron las dimensiones cognitiva, procesual y actitudinal.

\section{Tabla 1. Operacionalización de la variable}

\begin{tabular}{||l|l|}
\hline \multicolumn{1}{|c|}{$\begin{array}{l}\text { Variable: superación profesional técnica de los } \\
\text { profesores de la rama eléctrica }\end{array}$} \\
\hline 1.1 & $\begin{array}{l}\text { Dimensión 1: Cognitiva } \\
\text { a los avances científico-técnicos existentes } \\
\text { de la rama eléctrica }\end{array}$ \\
\hline 1.2 & $\begin{array}{l}\text { Nivel de renovación de los conocimientos y } \\
\text { habilidades profesionales de los avances } \\
\text { tecnológicos existentes en las entidades } \\
\text { laborales de la rama eléctrica }\end{array}$ \\
\hline 1.3 & $\begin{array}{l}\text { Nivel de profundización de conocimientos y } \\
\text { habilidades generales o específicas de la } \\
\text { rama eléctrica }\end{array}$ \\
\hline 1.4 & $\begin{array}{l}\text { Nivel de adquisición de nuevos } \\
\text { conocimientos y habilidades profesionales }\end{array}$ \\
\hline \multicolumn{1}{|c|}{ Dimensión 2: Procesual } \\
\hline 2.1 & $\begin{array}{l}\text { Nivel de correspondencia entre las } \\
\text { necesidades y problemas de superación }\end{array}$ \\
\hline
\end{tabular}




\begin{tabular}{|c|c|}
\hline & $\begin{array}{l}\text { profesional técnica y las acciones } \\
\text { planificadas dirigidas a los profesores de la } \\
\text { rama eléctrica }\end{array}$ \\
\hline 2.2 & $\begin{array}{l}\text { Nivel de correlación entre la planificación de } \\
\text { las formas organizativas de la superación } \\
\text { profesional técnica y los resultados de la } \\
\text { evaluación profesoral }\end{array}$ \\
\hline 2.3 & $\begin{array}{l}\text { Nivel de relación entre la teoría y la práctica } \\
\text { a partir de los avances tecnológicos de la } \\
\text { rama eléctrica }\end{array}$ \\
\hline 2.4 & $\begin{array}{l}\text { Nivel de satisfacción de los profesores de la } \\
\text { rama eléctrica con la superación profesional } \\
\text { técnica que recibe }\end{array}$ \\
\hline & Dimensión 3: Actitudinal \\
\hline 3.1 & $\begin{array}{l}\text { Nivel de disposición ante la superación } \\
\text { profesional técnica asociada a los avances } \\
\text { tecnológicos existente en las entidades } \\
\text { laborales de la rama eléctrica }\end{array}$ \\
\hline 3.2 & $\begin{array}{l}\text { Nivel de compromiso con la adquisición de } \\
\text { nuevos conocimientos y habilidades } \\
\text { profesionales de la rama eléctrica }\end{array}$ \\
\hline 3.3 & $\begin{array}{l}\text { Nivel de permanencia en la realización de } \\
\text { actividades prácticas que posibiliten la } \\
\text { renovación de los conocimientos y } \\
\text { habilidades profesionales de la rama } \\
\text { eléctrica }\end{array}$ \\
\hline 3.4 & $\begin{array}{l}\text { Nivel de observancia de valores ético- } \\
\text { profesionales durante el proceso de } \\
\text { superación profesional técnica }\end{array}$ \\
\hline
\end{tabular}

La dimensión cognitiva aprecia los contenidos conceptuales (conceptos, hechos y datos) y los procedimentales (habilidades, hábitos, destrezas y procedimientos), valora el saber y saber hacer.

La dimensión actitudinal aprecia los comportamientos y expresión emocional (actitudes y valores), valora el ser o el saber ser. $Y$ las valoraciones constantes del funcionamiento de la superación profesional técnica en un período de tiempo prefijado para la consecución de las metas $\mathrm{u}$ objetivos propuestos a partir de formas organizativas en el contexto de la entidad laboral, se concreta en la dimensión procesual.

\subsection{Interpretación de dimensiones e indicadores}

La dimensión cognitiva, expresa el desarrollo alcanzado por el profesor en la apropiación, actualización, complementación y reorientación del contenido técnico profesional (sistema de conocimientos y habilidades de la rama eléctrica) y de los métodos de trabajo tecnológicos propios de la actividad profesional, desde una perspectiva de colaboración, de interacción que pueden ser evaluados en la actuación actual y perspectiva en la entidad laboral. La dimensión cognitiva valora el saber y saber hacer.

El indicador 1.1, se refiere al dominio que muestran los profesores de la documentación técnica sobre la base de los fundamentos básicos de los equipos eléctricos, interpretación (observar, analizar, reflexionar y aplicar) gráfica y escrita de los circuitos eléctricos y electrónicos, simbología, planos, croquis y metodología de proyección tecnológica.

El indicador 1.2, se refiere a la actualización que manifiestan los profesores de los conocimientos y habilidades profesionales de los procesos en el campo electroenergético y son capaces de realizar las mediciones correspondientes de las magnitudes eléctricas con tecnología moderna y el montaje de dispositivos, aparatos y equipos eléctricos según el esquema de conexiones dado en condiciones modeladas.

El indicador 1.3, se refiere a la complementación que presentan los profesores de los conocimientos y habilidades profesionales no recibidos durante la carrera y que resultan necesarios para el mejor desempeño en el proceso de la ETP: caracterizar (observar, analizar y determinar los rasgos significativos) sobre la base de la generación distribuida, los requerimientos técnicos, los regímenes de funcionamiento y de realizar operaciones de instalación, mantenimiento y reparación en las subestaciones eléctricas y grupos electrógenos en condiciones modeladas.

El indicador 1.4, se refiere a la reorientación de nuevos conocimientos y habilidades profesionales mayormente ajenos a la carrera universitaria cursada en virtud de los procesos racionalizadores de desarrollo: caracterizar, describir (observar, analizar, determinar y expresar los rasgos significativos) y realizar producciones energéticas sostenibles en condiciones modeladas: tecnología de producción de Biogás, entre otras.

La dimensión procesual, expresa el reconocimiento de potencialidades y dificultades del proceso de superación profesional técnica, donde luego de una situación inicial, se pretende el establecimiento de cambios permanentes y eficaces en los profesores, consta de las valoraciones constantes del funcionamiento de la superación profesional técnica en un período de tiempo prefijado para la 
consecución de las metas u objetivos propuestos. La dimensión procesual valora el ajustar y regular sobre la marcha el proceso de superación profesional técnica.

El indicador 2.1, se refiere a la armonía de las acciones planificadas en las estrategias de superación, los planes de desarrollo individual y las necesidades y problemas de superación profesional técnica de los profesores.

El indicador 2.2, se refiere a la correspondencia existente entre la planificación de formas organizativas de superación profesional técnica, teniendo en cuenta los resultados del diagnóstico y las necesidades y problemas individuales de los profesores revelados en la evaluación profesoral.

El indicador 2.3, se refiere al paralelismo entre las actividades de carácter teórico-práctico concebidas para la actualización de los profesores en los conocimientos y habilidades profesionales de los procesos en el campo electroenergético.

El indicador 2.4, se refiere a la complacencia de los profesores de la rama eléctrica con la superación profesional técnica que recibe para la adquisición de nuevos conocimientos y habilidades profesionales en las entidades laborales.

La dimensión actitudinal, expresa el compromiso, la constancia y la disposición del profesor para responder a la superación profesional técnica, el saber actuar y convivir en espacios o escenarios definidos para ello, y el cumplimiento de valores ético-profesionales. La dimensión actitudinal valora el ser o el saber ser.

El indicador 3.1, se refiere a la inclinación afectiva de los profesores para la actualización de los conocimientos y habilidades profesionales de la rama eléctrica asociado a los avances tecnológicos existente en las entidades laborales.

El indicador 3.2, se refiere al empeño manifestado para la adquisición de nuevos conocimientos y habilidades profesionales de la rama eléctrica para la transformación de sí mismo, de su entorno social y profesional.

El indicador 3.3, se refiere a la constancia que expresan los profesores en la realización de actividades prácticas que posibiliten la renovación sistemática de los conocimientos y habilidades profesionales de la rama eléctrica.
El indicador 3.4, se refiere al cumplimiento de valores ético-profesionales durante la adquisición de nuevos conocimientos y habilidades profesionales de la rama eléctrica (la responsabilidad, la laboriosidad, el cuidado de la propiedad social y el medio ambiente).

\subsection{Diagnóstico de la superación profesional técnica de los profesores de la rama eléctrica}

Sobre los años de experiencia de los profesores de la rama eléctrica y del personal directivo, se destaca como aspecto positivo que el $72 \%$ del personal directivo posee vasta experiencia en el subsistema de la ETP (de 11 a 15 años de experiencia). Al igual que entre un $40 \%-50 \%$ de los profesores de la rama eléctrica poseen de 6 a10 años de experiencia, todo lo cual es favorable en la obtención de la información pertinente para identificar las necesidades y problemas de superación profesional técnica y enrumbar la proyección hacia la solución inmediata de problemas apremiantes.

Los resultados de la revisión documental revelan que se han diseñado y realizado varias acciones de superación pero no con un enfoque profesional técnico de la rama eléctrica que responda a las recomendaciones de la evaluación profesoral, por lo que solo han dado solución parcial a las necesidades y problemas de los profesores en un momento determinado y está deprimida la apropiación de los conocimientos y habilidades del contenido técnico profesional de la rama eléctrica desde las formas organizativas de superación.

De la triangulación de los instrumentos aplicados, los resultados del estado de los indicadores al inicio revelan que de los doce indicadores medidos, resultan menos favorecidos los ocho indicadores correspondientes a las dimensiones cognitiva y procesual, pues no alcanzan las tendencias centrales y los valores oscilan entre (1,9 hasta 2,84), sin embargo los indicadores de la dimensión actitudinal revelan valores desde $(3,93$ hasta 4,89 ) los que resultan los más favorecidos, todo lo cual constituye una fortaleza, y se evidencia en la disposición y el nivel de compromiso de los profesores con la adquisición de nuevos conocimientos y habilidades profesionales de la rama eléctrica.

Los resultados del estado de las dimensiones al inicio para los diferentes estratos infieren la existencia de insuficiencias en el proceso de 
superación profesional técnica. Sin embargo, un análisis relativo, puede evidenciar ciertas diferencias entre las dimensiones. El comportamiento de las dimensiones se presenta en la tabla 2. Y el grado de coherencia y objetividad de los resultados obtenidos a partir del coeficiente de correlación multidimensional rpj permiten inferir con un alto grado de probabilidad, que se realizaron análisis objetivos y por tanto, confiables. Los resultados se presentan en la tabla 3.

Tabla 2. Comportamiento de las tendencias centrales de las dimensiones en la triangulación

\begin{tabular}{|c|c|c|c|}
\hline Instrumentos & $\begin{array}{c}\text { Dimensión } \\
\text { Cognitiva }\end{array}$ & $\begin{array}{c}\text { Dimensión } \\
\text { Procesual }\end{array}$ & $\begin{array}{c}\text { Dimensión } \\
\text { Actitudinal }\end{array}$ \\
\hline $\begin{array}{c}\text { Guía de } \\
\text { observación }\end{array}$ & 1,90 & 1,96 & 2,75 \\
\hline $\begin{array}{c}\text { Cuestionario } \\
\text { a directivos }\end{array}$ & 1,73 & 1,99 & 2,28 \\
\hline $\begin{array}{c}\text { Cuestionario } \\
\text { a profesores }\end{array}$ & 2,79 & 2,78 & 3,85 \\
\hline $\begin{array}{c}\text { Estado de } \\
\text { las } \\
\text { dimensiones }\end{array}$ & 1,9 & 1,99 & 2,75 \\
\hline
\end{tabular}

Triangulación. Estado de las dimensiones al inicio

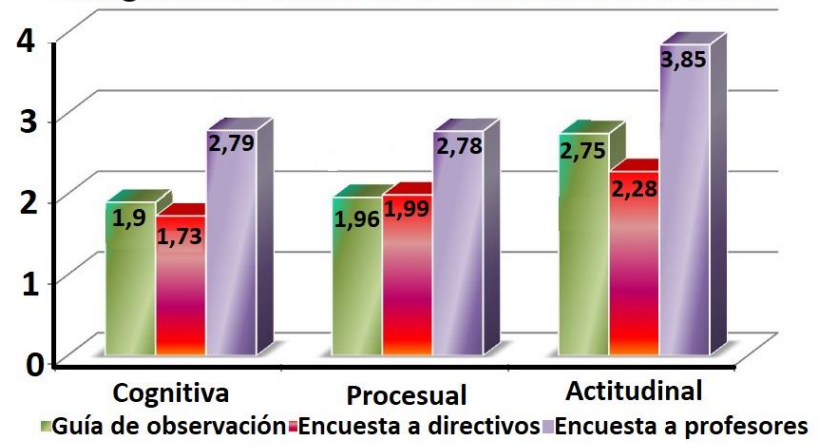

Gráfico 1: Comportamiento de las tendencias centrales de las dimensiones en la triangulación.

\section{Estado de cada dimensión en la variable}

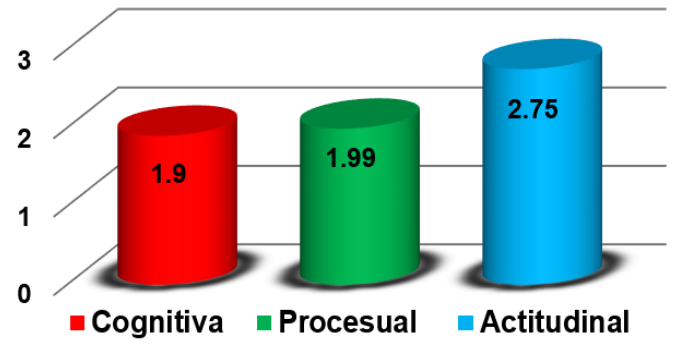

Variable: Superación profesional técnica de los profesores de la rama eléctrica
Gráfico 2: Comportamiento de la variable: superación profesional técnica de los profesores de la rama eléctrica en la etapa inicial

Tabla 3. Resultado del coeficiente de correlación multidimensional rpj para cada dimensión

\begin{tabular}{|l|c|c|c|}
\hline Resultado & Cognitiva & Procesual & Actitudinal \\
\hline Coeficiente & 0,970 & 0,968 & 0,961 \\
\hline Criterio & $\begin{array}{l}\text { Altamente } \\
\text { coherente }\end{array}$ & $\begin{array}{c}\text { Altamente } \\
\text { coherente }\end{array}$ & $\begin{array}{c}\text { Altamente } \\
\text { coherente }\end{array}$ \\
\hline
\end{tabular}

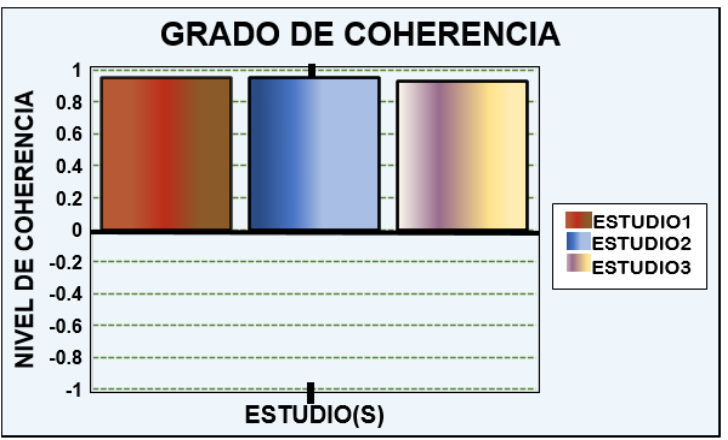

Gráfico 3: Comportamiento del coeficiente de correlación multidimensional rpj para cada dimensión

\section{Conclusiones}

La sistematización teórica realizada acerca del proceso de superación profesional técnica en la ETP, permitió establecer los rasgos que lo distinguen y lo caracterizan en la rama eléctrica como un proceso pedagógico que ejecutan los profesores, a partir de la actualización, complementación y reorientación de sus conocimientos, habilidades técnico-profesionales, actitudes y valores, para obtener los resultados en correspondencia con las exigencias actuales y los roles que desempeñan.

El diagnóstico del estado inicial de la superación profesional técnica de los profesores de la rama eléctrica de los institutos politécnicos en la provincia de Artemisa evidenció que el comportamiento de las dimensiones se ubican en el rango evaluativo de bajo, alejado de la tendencia central: dimensión cognitiva (1,90); dimensión procesual $(1,99)$ y dimensión actitudinal $(2,75)$. Se reconocen insuficiencias en la articulación entre la universidad, las direcciones municipales y provinciales de Educación, los institutos politécnicos y las entidades laborales para la actualización, complementación y reorientación del contenido técnico profesional de los profesores de la rama eléctrica, pues no se aprovechan las 
potencialidades del entrenamiento como forma de organización.

\section{Agradecimientos}

Al Dr. C. Omar Pérez Jacinto por su ciencia, exigencia e imprescindible colaboración. A los profesionales del Departamento de Electricidad del Instituto Politécnico "Juan Manuel Castiñeiras" y a los de la Termoeléctrica "Máximo Gómez" cuya ayuda ha sido de incuestionable valor. A todos los que hicieron posible este trabajo, muchas gracias.

\section{Referencias}

[1] A. Rodríguez, y O. A. Pérez. Métodos científicos de indagación y construcción de conocimientos. Revista EAN 82 (2017) 175195, Visitado el 14/6/2017. Disponible en :

https://doi.org/10.21158/01208160.n82.2017. 1647

[2] O. Pérez, T. Crespo, I. Arnaez, R. Hernández Los diseños estadísticos en las investigaciones educativas (Educación Cubana, Ciudad de la Habana, 2011). Visitado el 22/6/2017. Disponible en : http://www.cubaeduca.cu/media/www.cubaed uca.cu/medias/pdf/2878.
[3] J. Paul, Between Method Triangulation in Organizational Diagnosis. The International Journal of Organizational Analysis 4 (1996) 135-153. Disponible en: https://doi.org/10.1108/eb028845

[4] M. Fernández, Tesis de Doctorado, Facultad de Ciencias Técnicas, Universidad de Pinar del Río,2014.

[5] Z. López, Tesis de Doctorado, Facultad de Ciencias Técnicas, Universidad de Ciencias Pedagógicas Enrique José Varona, 2015. Disponible en: https://dialnet.unirioja.es/descarga/tesis/1219 $\underline{59 . p d f}$

[6] O. Pérez, T. Crespo, I. Arnaez, y R. Hernández, Los diseños estadísticos en las investigaciones educativas (Educación Cubana, Ciudad de la Habana, 2011), p.17. Visitado el 22/6/2017.

Disponible en: http://www.cubaeduca.cu/media/www.cubaed uca.cu/medias/pdf/2878.

E-mail: zeidysandra@uart.edu.cu, zlopezcollazo@gmail.com 